\title{
Predição por calibração multivariada dos parâmetros de qualidade de biomassas de café
}

\author{
Prediction by multivariate calibration of quality parameters of coffee residues
}

\author{
Magale Karine Diel Rambo ${ }^{*}$; Luana Priscilla Rodrigues Macêdo ${ }^{2}$; Marcleane Mendes da Silva ${ }^{3}$ \\ e Michele Cristiane Diel Rambo
}

\author{
${ }^{1}$ Doutora, Universidade Federal do Tocantins- UFT, Araguaína- Brasil \\ ${ }^{2}$ Aluna de Iniciação científica, Universidade Federal do Tocantins- UFT, Araguaína- Brasil \\ ${ }^{3}$ Aluna de Iniciação científica, Universidade Federal do Tocantins- UFT, Araguaína- Brasil \\ ${ }^{4}$ Mestre, Instituto Federal de Educação, Ciência e Tecnologia do Tocantins- IFTO, Palmas, Brasil.
}

\begin{abstract}
Resumo
A previsão em tempo real dos parâmetros de qualidade das biomassas de café permite uma maior eficácia nas indústrias e no seu processo de produção de energia, permitindo a determinação de múltiplos constituintes químicos e evitando simultaneamente a extensa preparação da amostra. Modelos de calibração multivariada utilizando espectros obtidos pelo infravermelho próximo (NIR) foram desenvolvidos e aplicados para prever as seguintes propriedades do café: umidade, cinzas, carbono fixo e teor de voláteis. Resultados satisfatórios foram obtidos demonstrando a importância da metodologia sob o ponto de vista econômico, mas também ambiental, já que resíduos da indústria de café (cascas) foram avaliados e demonstraram potencial de aproveitamento.
\end{abstract}

Palavras-chave: Café. Análise aproximada. Espectroscopia NIR. Quimiometria

\begin{abstract}
The real time prediction of the quality of the coffee biomass parameters allow greater efficiency in the energy industry and its production process, allowing the determination of multiple chemical components, while avoiding the extensive sample preparation. Multivariate calibration models using spectra obtained by near infrared spectroscopy (NIR) have been developed to predict the following properties of coffee: moisture, ash, fixed carbon and volatile content. Satisfactory results were obtained demonstrating the importance of methodology from the economic point of view, but also environmental, since residues from the coffee industry (husks) were evaluated and demonstrated use of potential.
\end{abstract}

Keywords: : Coffee. Ultimate analysis. NIR spectroscopy. Chemometrics 


\section{Introdução}

$\mathrm{D}$ urante a última década, a análise de espectroscopia no infravermelho próximo (NIR) surgiu como uma ferramenta analítica confiável e promissora para a avaliação da qualidade dos atributos do café, como também de outras biomassas (Gillespie et al., 2015; Barbin et al., 2014; Rambo et al.,2013). A espectroscopia NIR tem um enorme potencial para a obtenção de informações rápidas sobre a composição química do café, além da capacidade de quantificar algumas características importantes, tais como umidade, cinzas, teor de carbono fixo, material volátil entre inúmeros outros.

Desenvolver um sistema de avaliação da qualidade e além do mais rápido com base em informação espectral infravermelha para avaliar os parâmetros de qualidade do café traz benefícios econômicos para a indústria, como também para o consumidor.

Esse grande interesse da indústria do café em tecnologias cada vez mais rápidas e eficazes está relacionado ao fato do café ser a segunda bebida mais popular do mundo, e uma das matérias-primas mais importantes dentro do comércio internacional (Barbin et al., 2014). Sem contar que seu aproveitamento não está relacionado apenas ao consumo do grão, mas também a outras importantes atividades, principalmente quando se pensa no aproveitamento de seus resíduos, como as cascas de café. Agregar valor a resíduos fornecendo energia tem sido um dos assuntos mais discutidos, diante da atual crise energética que o Brasil e o mundo vêm enfrentando. A implantação de biorrefinarias aproveitando tais resíduos e os transformando em produtos, tem atraído interesse do mundo todo (Krzyzaniak et al., 2014; Kiran et al., 2014). Principalmente quando se trata de uma indústria com alta produção de biomassa residual, tal qual a do café, onde para cada $1 \mathrm{~kg}$ de frutos de café produzidos, aproximadamente $0,18 \mathrm{~kg}$ de cascas são geradas (Murthy e Naidu, 2012).

É sabido que o Brasil é o maior produtor de café com uma produção de 27,5 milhões de sacas (de $60 \mathrm{~kg}$ ), dos 146 milhões de sacas produzidos mundialmente, segundo dados da Organização Internacional do Café (ICO, 2014). Dessa forma uma utilização adequada para esses resíduos precisa ser criada.

Avaliar a qualidade dos resíduos de café de forma rápida, de baixo custo e confiável tem sido possível graças a união da espectroscopia NIR com a quimiometria. Essa aliança se dá através da construção de modelos de calibração multivariada, o que minimiza as análises por via úmida, uma vez já obtidos os dados de referência. Essa grande atenção tem sido atribuída à possibilidade de se realizarem análises diretas, sem necessidade de pré-tratamento das amostras, de ser uma técnica não destrutiva e rápida, obtendo-se resultados em intervalos de tempo da ordem de segundos. O grande inconveniente associado trata da difícil interpretação dos espectros NIR. No entanto esse problema pode ser contornado tratando os dados espectrais com o uso de técnicas quimiométricas obtendo-se assim resultados precisos e confiáveis a partir das informações contidas nos espectros NIR (Rambo et al., 2013). Ambos, quimiometria e a espectroscopia NIR evoluíram em uma espécie de simbiose, ou seja, uma relação mutuamente vantajosa, que permite obter mais informação da espectroscopia com possibilidade de gerar modelos de quantificação confiáveis (Pasquini, 2003).

\section{Material e Métodos}

\subsection{Amostras}

Trinta e cinco amostras de cascas de café, distribuídas entre as espécies arábica e robusta de diversos cultivares (mundo novo, caturra, catuaí, conilon), fornecidas por empresa beneficiadora- IAC (Instituto Agronômico de Campinas) foram devidamente coletadas. Essas amostras foram mantidas congeladas em sacos plásticos até as etapas seguintes do processo.

Todas as amostras coletadas foram secas em temperatura ambiente. Em seguida as amostras foram moídas em um moinho tipo faca (MA 920, Marconi) e classificadas em agitador de peneiras automático (VP-01, Bertel) para análises granulométricas e para redução do tamanho de partícula. Foram utilizadas as frações secas e moídas com partículas de diâmetro entre $180 \mu \mathrm{m}$ e $850 \mu \mathrm{m}$ para os trabalhos de investigação pelos métodos de referência via úmida (análise química aproximada).

\subsection{Análise química aproximada}

Os procedimentos utilizados para a análise química aproximada, ou imediata como também é conhecida, foram realizados segundo as normas da Sociedade Americana de Testes e Materiais (ASTM).

A umidade foi determinada (ASTM D 3173-87) após a amostra ser aquecida a $105^{\circ} \pm 5^{\circ} \mathrm{C}$ em estufa (SP 100, SP Labor) durante 12 horas, ou até obter massa constante. O teor de umidade foi calculado da seguinte forma:

$$
\text { Umidade }(\%)=\frac{\mathrm{Mi}-\mathrm{Mv}}{\mathrm{Mi}} \times 100 \quad \text { Equação } 1
$$

onde Mi é a massa em gramas da amostra usada;

Mv é a massa da amostra em gramas após aquecimento.

O teor de matérias voláteis (TMV) foi determinado (ASTM D 3175 - 07) usando 1 grama da amostra, previamente seca, em mufla (1200DRP7, SP Labor) a 800 $\pm 10 \stackrel{\circ}{\circ} \mathrm{C}$ por 8 minutos. A amostra foi retirada e colocada em dessecador para ser resfriada, durante 60 minutos, sendo finalmente pesada e seu teor de TMV calculado de acordo com a equação 2:

$$
\mathrm{CZ}(\%)=\frac{M v \times 100}{M i}
$$


onde Mi: é a massa em gramas da amostra usada;

Mv: é a massa da amostra em gramas após aquecimento.

O teor de cinzas (CZ) foi determinado de acordo com a ASTM (D 3174 - 04) que envolveu a remoção de constituintes orgânicos através de altas temperaturas em mufla (1200DRP7, SP Labor) por um período de 4 horas a $600 \pm 10^{\circ} \mathrm{C}$. O CZ (\%) foi determinada de acordo com a equação 3:

$$
\operatorname{TMV}(\%)=\frac{\mathrm{Mi}-\mathrm{Mv}}{\mathrm{Mi}} \times 100
$$

Equação 3

onde Mv: é a massa da amostra após oxidação em mufla; Mi: é a massa em gramas da amostra usada;

O teor de carbono fixo (CF) é obtido de forma indireta e foi calculado pela equação 4:

$$
\mathrm{CF}(\%)=100-(C Z+T M V)
$$

Equação 4

Para a análise química aproximada, critérios foram definidos de modo a assegurar a precisão do método de referência. Esses foram baseados no desvio padrão das duplicatas. Todas as análises foram realizadas em duplicata, com desvio padrão inferiores a $1 \%$. No caso de resultados com desvio padrão superiores a $1 \%$, as análises foram repetidas.

\subsection{Espectroscopia no Infravermelho-próximo (NIR)}

Os espectros na região do infravermelho próximo foram obtidos em um espectrômetro FOSS (XDS FOSS, Hillerød) de feixe único, no modo de reflectância difusa, na faixa de 400 a $2500 \mathrm{~nm}$ (completo). Os dados de reflectância $(\mathrm{R})$ foram transformados para absorbância (A) usando $A=\log _{10}(1 / R)$. Cada espectro foi obtido pela média de 32 varreduras, com incremento de $0,5 \mathrm{~nm}$. Cada fração de amostra foi analisada em duplicata, e então o espectro médio foi utilizado para análise posterior.

\subsection{Análise dos Dados}

As análises estatísticas e multivariadas foram realizadas utilizando os softwares Unscrambler 10.3 para construção dos modelos de calibração multivariada e Origin 6.0 (Originlab) para construção dos gráficos e figuras ilustrativas.

Para os modelos preditivos dos componentes químicos foi utilizado o método de regressão por quadrados mínimos parciais (PLS1), no qual as variáveis (analitos de interesse) são analisados separadamente (Geladi e Kowalski, 1986). É um método de regressão linear que relaciona os dados espectrais $\mathbf{X}$ à propriedade de interesse $\mathbf{y}$ (por exemplo, umidade, cinzas, carbono fixo). A propriedade de interesse $\mathbf{y}$ (I x 1), também conhecida como variável predita, é obtida experimentalmente por meio de padrões certificados ou algum método de referência. A covariância entre os escores e o vetor y é maximizada, ou seja, a informação de y é incorporada e se calcula a matriz de pesos (W) de dimensão ( $\mathbf{J} \times \mathbf{~ m})$, onde $\mathbf{J}$ é o número de variáveis preditoras (no caso do NIR as variáveis são os comprimentos ou números de onda) e m o número de fatores selecionados, denominados de variáveis latentes. Os escores expressam as relações entre as amostras enquanto que os pesos mostram as relações entre as variáveis originais.

Diferentes pré-processamentos foram testados, e os melhores resultados foram obtidos com a segunda derivadas (2D) usando o tratamento Savitzky Golay (Savitzky e Golay, 1964). No uso das derivadas, variou-se o tamanho das janelas, usando de 3 até 31 pontos, sendo que o valor empregado (3 pontos) resultou no maior ganho na relação sinal/ruído. O polinômio usado foi sempre de ordem 2 para todos os modelos.

A validação dos modelos foi realizada utilizando a validação cruzada (Martens e Naes, 1996), no caso de conjuntos com pequenas quantidades de amostras $(\mathrm{N}<40)$.

Modelos utilizando o método PLS foram construídos para diferentes analitos que foram: umidade; cinzas; CF e TMV.

Os espectros foram utilizados considerando-se apenas a região do NIR (1100 a $2500 \mathrm{~nm}$ ). A fim de facilitar a construção dos modelos de calibração o método de seleção de variáveis foi empregado, com base nas regiões características dos analitos.

Os modelos de regressão obtidos foram avaliados de acordo com os seguintes parâmetros: coeficiente de determinação da calibração $\left(\mathrm{R}_{\text {cal }}^{2}\right)$, coeficiente de determinação da validação cruzada $\left(\mathrm{R}^{2}{ }_{\mathrm{cv}}\right)$, raiz quadrada do erro quadrático médio de calibração (RMSEC), raiz quadrada do erro quadrático médio de validação cruzada (RMSECV), número de variáveis latentes (VL), fração de amostras anômalas excluídas, erro relativo $(\mathrm{ER} \%=\mathrm{RMSECV} * 100 /$ média dos valores estimados $)$. As equações utilizadas para o cálculo dos parâmetros estatísticos foram as propostas por Rambo et al. (2013).

\section{Resultados e Discussões}

Os resultados obtidos para os modelos construídos com o método PLS são mostrados na Tabela 1.

Para a umidade, apesar de o analito ser um composto único os espectros NIR apresentam várias bandas significativas para a água. Isso ocorre devido à existência de diferentes interações moleculares entre a água e moléculas adjacentes da biomassa (Lestander e Geladi, 2005), necessitando assim de 2 VL para ser corretamente modelada. Os modelos obtidos aqui apresentaram $\mathrm{R}_{\text {cal }}^{2}$ de 0,96 e $R_{\text {cv }}^{2}$ de 0,88 .

$\mathrm{O}$ que ocorre no caso das cinzas é que a espectroscopia NIR se limita a substâncias orgânicas. Entretanto, a tentativa de criação de modelos para o teor de cinzas (substâncias inorgânicas) se justifica, pois, esses compostos podem se correlacionar com outros compostos químicos que absorvem no NIR, e assim por "correlação indireta" serem modelados (Lestander e Rhen, 2005). Esse fato foi observado nesse trabalho com valores satisfatórios de $\mathrm{R}_{\text {cal, cv }}^{2}$ e baixo ER.

Para o carbono fixo, como o analito alvo é um com- 
Tabela 1 - Resultados dos modelos de calibração PLS obtidos

\begin{tabular}{l|c|c|c|c|c|c|c|c}
\hline Analito & $\begin{array}{l}\text { Tamanho da } \\
\text { matriz }\end{array}$ & VL & $\begin{array}{l}\text { Amostras } \\
\text { anômalas }\end{array}$ & $\begin{array}{c}\mathrm{R}^{2} \\
\mathrm{cal}\end{array}$ & $\begin{array}{c}\mathrm{R}^{2} \\
\mathrm{c}_{\mathrm{V}}\end{array}$ & RMSEC & RMSECV & ER \\
\hline Umidade & $41 \times 93$ & 2 & - & 0,96 & 0,86 & 0,455 & 0,881 & 8,10 \\
\hline Cinzas & $40 \times 112$ & 2 & 1 & 0,96 & 0,80 & 0,065 & 0,157 & 7,60 \\
\hline CF & $40 \times 83$ & 1 & 1 & 0,87 & 0,81 & 1,100 & 1,328 & 12,00 \\
\hline TMV & $39 \times 35$ & 2 & 2 & 0,86 & 0,76 & 1,331 & 1,820 & 2,10 \\
\hline
\end{tabular}

posto único constituído apenas de carbono, $1 \mathrm{VL}$ foi suficiente para fornecer bons modelos de calibração. $\mathrm{O}$ ER foi o maior dentre todos os parâmetros analisados, mas considerando que apenas uma amostra anômala foi removido e 1 VL utilizada, o modelo se mostrou satisfatório.

Para o TMV 2 VL foram necessárias na modelagem. Isso pode ser explicado pelo fato de que os materiais voláteis são uma mistura de inúmeros compostos que provavelmente absorvem em diferentes regiões do espectro, e assim, justificam a necessidade de duas variáveis para explicar a variância associada. $\mathrm{O}$ valor de $\mathrm{R}_{\mathrm{cv}}^{2}$ encontrado foi inferior a 0,80 e duas amostras tiveram de ser removidas para um bom ajuste do modelo. No entanto o modelo não parece ter sido prejudicado dado o seu baixo ER (2,10\%).
As Figuras 1 (A-D) mostram as respectivas curvas de calibração e validação cruzada, para os modelos de TMV, $\mathrm{CF}$, cinzas e umidade, respectivamente. As concordâncias entre os valores de referência e os valores previstos, tanto para os conjuntos de calibração e de validação cruzada indicam que os modelos são bons. A figura 1 (A) apresenta valores de TMV na faixa de 74,80-92,7\% indicando que as cascas de café podem ser bem aproveitadas na combustão para a produção de energia. $\mathrm{O}$ teor de CF (Figura 1B) ficou no intervalo de 5,50-20,12\% o que demonstra a possibilidade das amostras com teores mais elevados de CF serem aproveitadas na produção de carvão vegetal (Santos et al., 2012). O gráfico de cinzas (Figura 1C) compreende valores baixos na faixa de 1,35$2,93 \%$, o que agrega valor a essas biomassas de café, já que um elevado teor de cinzas atrapalha nos processos
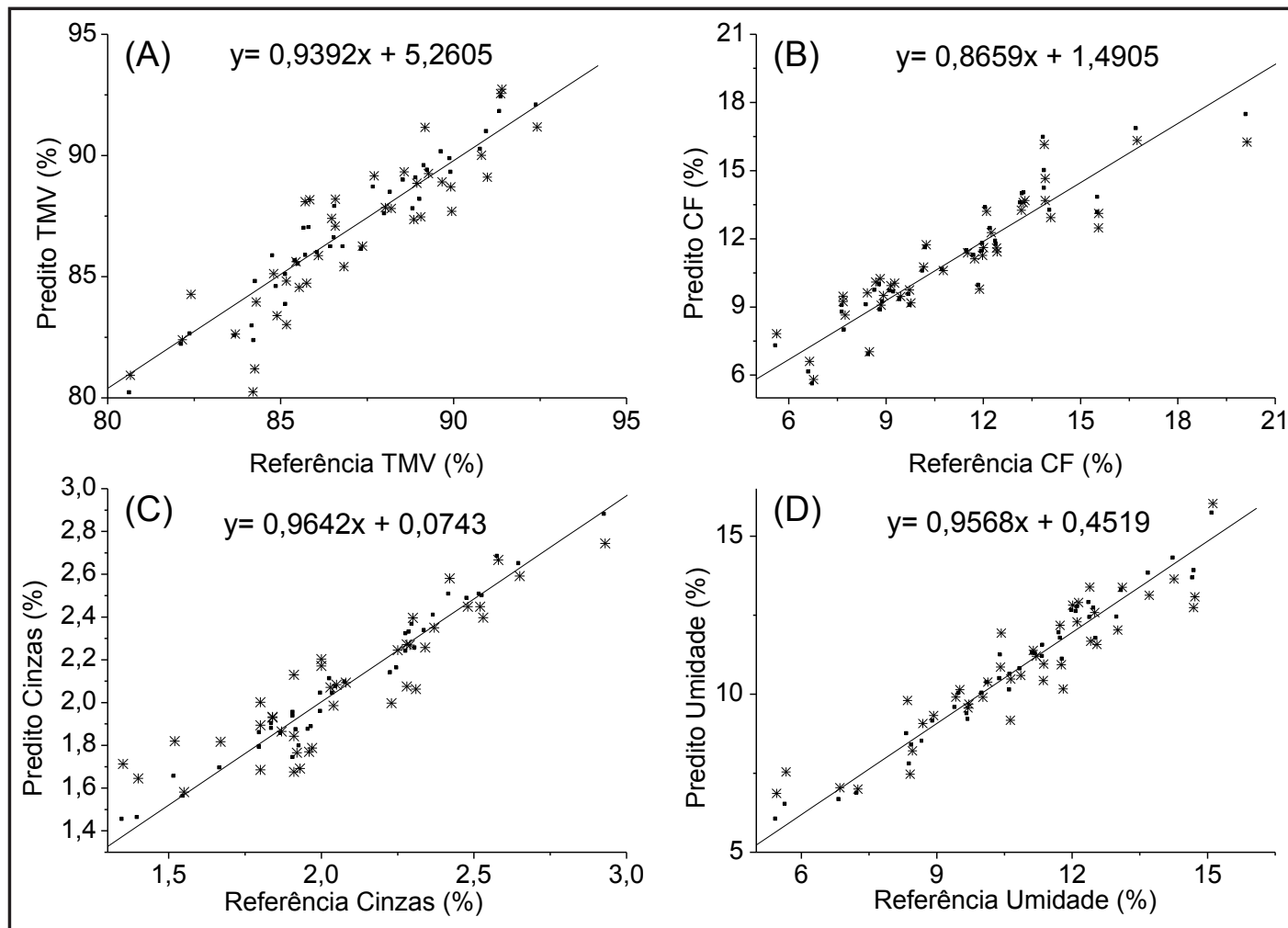

Figura 1 - Curvas de calibração e validação cruzada dos valores de referência versus os valores preditos para (A) Teor de matéria volátil (TMV) com 2 VL; (B) Carbono fixo (CF) com 1VL; (C) Cinzas com 2 VL; (D) Umidade com 2 VL 


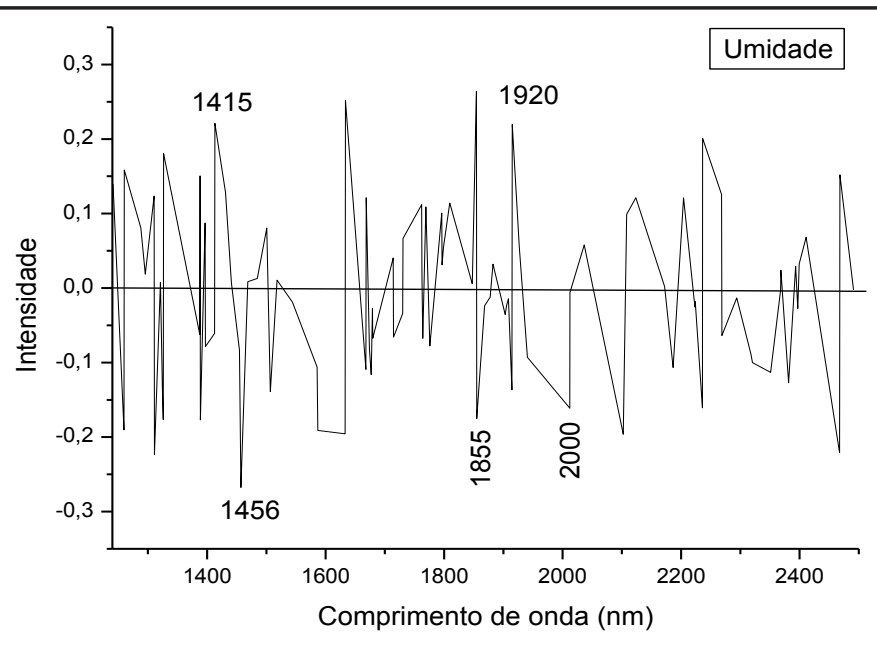

(A)

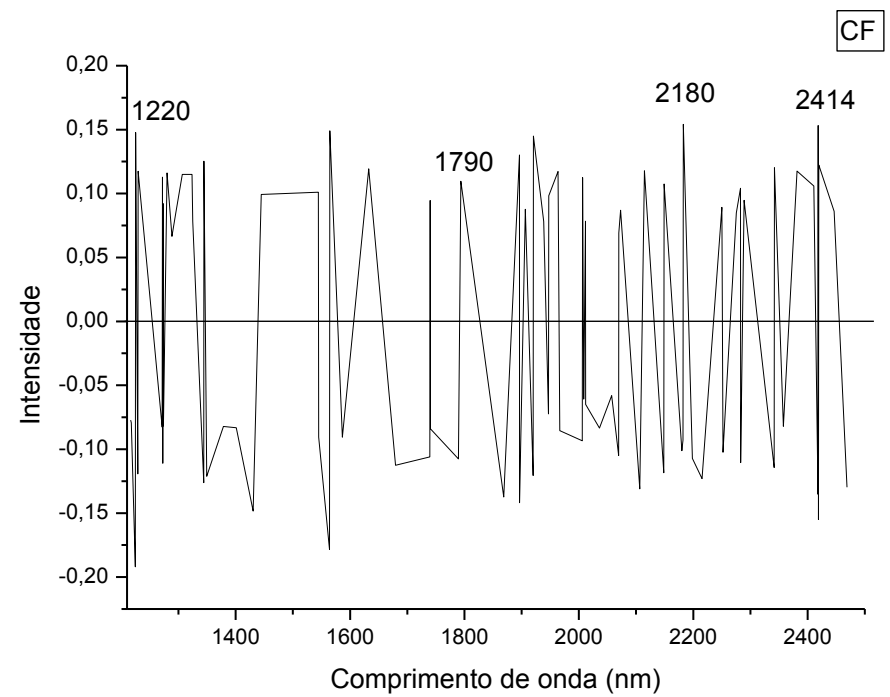

(B)

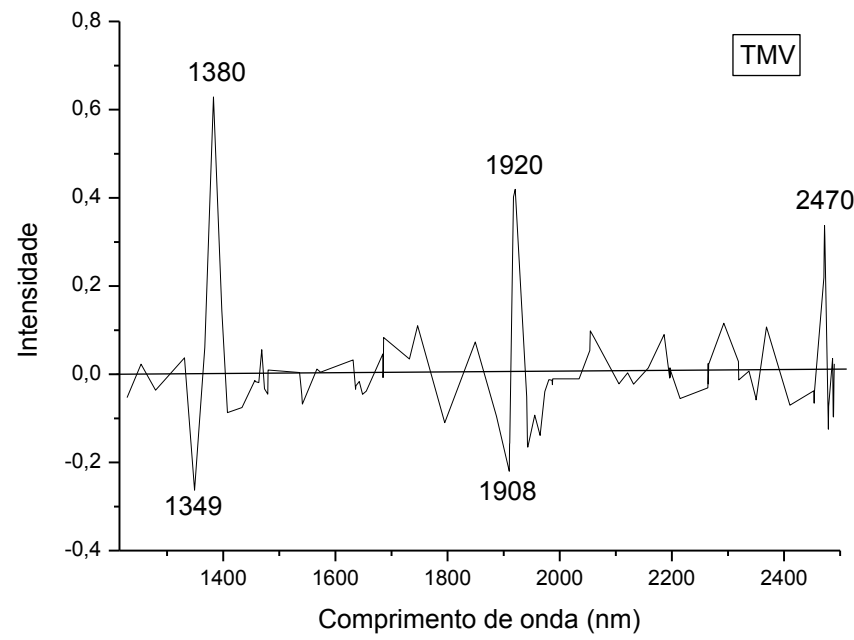

(C)

Figura 2 - Coeficientes de regressão para os modelos PLS de (A) umidade; (B) carbono fixo e (C) teor de matéria volátil. 
de produção de energia (Bruun et al., 2010). Os teores de umidade foram todos inferiores a 16\% (Figura 1D), fator também favorável para os processos de combustão (Everard et al., 2012).

A fim de assegurar a confiabilidade desses modelos PLS, coeficientes de regressão foram interpretados como forma de garantir que o analito modelado tenha relação com a curva de calibração obtida (Figura 2).

Os coeficientes de regressão para a umidade (Figura 2A) apresentam bandas típicas de água (1920 nm e $1415 \mathrm{~nm}$ ) (Shenk et al., 2008). As regiões negativas correlacionadas com a umidade foram principalmente ácidos carboxílicos (1855 nm), que juntamente com o estiramento alquil de primeiro sobretom, podem ser atribuídas a ácidos graxos, componentes hidrofóbicos. Proteínas (1456 nm) e celulose (2000 nm) (Ali et al., 2001) também apresentam uma correlação negativa com o teor de umidade.

Coeficientes de regressão para o CF (Figura 2B) apresentam bandas típicas de ligações $\mathrm{C}-\mathrm{H}$ de segundo sobretom (1220 nm); estiramento C-H de primeiro sobretom (1790 nm) oriundo provavelmente de celulose; bandas típicas de combinação de C-C em $2180 \mathrm{~nm}$ e de C-H em 2414 nm (Shenk et al., 2008).

Já para o TMV (Figura 2 C) bandas em 1380 nm, 1920 nm e 2470 nm tiveram relação direta com o TMV, e correspondem respectivamente ao estiramento de $\mathrm{Ar}-\mathrm{OH}$, estiramento $\mathrm{O}-\mathrm{H}$ de água e bandas de combinação $\mathrm{C}-\mathrm{H}$, todos associados a substâncias voláteis. Para cinzas não faz sentido a interpretação dos coeficientes de regressão, uma vez que a correlação provavelmente é indireta, como já citado anteriormente.

\section{Conclusões}

Os resultados satisfatórios dos modelos de calibração multivariada sugerem que a espectroscopia NIR tem potencial na análise aproximada de biomassas de café em processos rápidos, baratos e sem laboriosas etapas de preparação uma vez que apresentaram baixos \%ER e coeficientes de determinação superiores a 0,80 .

Além do mais a utilização de resíduos como forma de aproveitamento para a obtenção de energia é muito importante para uma sociedade economicamente viável e ambientalmente correta.

\section{Agradecimentos}

Ao CNPq (420154/2013-2) pelo apoio financeiro, e ao IAC pelo fornecimento das amostras.

\section{Referências}

Ali, M.; Emsley, A.M.; Herman, H.; Heywood, R.J.
Spectroscopic studies of the ageing of cellulosic paper. Polym. 42 (2001) 2893-2900.

ASTM D 3173-87. Standard method for determination of moisture content in biomass; 2003.

ASTM D 3174-04. Standard method for ash in the analysis sample of coal and coke; 2004.

ASTM D 3175-07. Standard method for volatile matter in the analysis sample of coal; 2007.

Barbin, D.F.; Felicio, A.L.S.M.; WenSunb, D.; Nixdorf, S.L.; Hirooka, E.Y. Application of infrared spectral techniques on quality and compositional attributes of coffee: An overview. Food Res. Int. 61 (2014) 23-32.

Bruun, S.; Jensen, J.W.; Magid, J.; Lindedam, J.; Engelsen, S.B. Prediction of the degradability and ash content of wheat straw from different cultivars using near infrared spectroscopy. Ind. Crop Prod. 31 (2010) 321-326.

Everard, C. D.; McDonnell, K.P.; Fagan, C.C. Prediction of biomass gross calorific values using visible and near infrared spectroscopy. Biomass Bioenerg. 45 (2012) 203-211.

Geladi, P.; Kowalski, B.R. Partial least squares regression: A tutorial. Anal. Chim. Acta. 185 (1986) 1-17.

Gillespie, G.D.; Everard, C.M.; McDonnell, K.P. Prediction of biomass pellet quality indices using near infrared spectrocopy. Energy 80 (2015) 582-588.

ICO. International Coffee Organization. Disponível em http://www.ico.org/. Acessado: Janeiro/2013.

Kiran, E.U.; Trzcinski, A.P.; Jern Ng, W.; Liu,Y. Bioconversion of food waste to energy: A review. Fuel 134 (2104) 389-399.

Krzyzaniak,M.; Stolarski, M.J.; Waliszewska,B.; Szczukowski, S.; Tworkowski, J.; Załuski, D.; Snieg, M. Willow biomass as feedstock for an integrated multi-product biorefinery. Ind. Crop Prod. 58 (2014) 230-237

Lestander, T. A.; Rhen, C. Multivariate NIR spectroscopy models for moisture, ash and calorific content in biofuels using bi-orthogonal partial least squares regression. Analyst 130 (2005) 1182-1189.

Lestander, T. A.; Geladi, P. NIR spectral information used to predict water content of pine seeds from multivariate calibration. Can. J. For. Res. 35 (2005) 1139-1148. 
MARTENS, H.; NAES, T. Multivariate Calibration. John Wiley e Sons, New York, 1996.

Murthy, P.S.; Naidu, M.M. Sustainable management of coffee industry by-products and value addition - A review. Resour. Conserv. Recy. 66 (2012) 45-58.

Pasquini, C. Near Infrared Spectroscopy: Fundamentals, Practical Aspects and Analytical Applications. J. Braz. Chem. Soc. v. 14, p.198-219, 2003.

Rambo, M.K.D; Amorim, E.P; Ferreira, M.M.C. Potential of visible-near infrared spectroscopy combined with chemometrics for analysis of some constituents of coffee and banana residues. Anal. Chim. Acta 775 (2013) 41-49.

Santos, R.C.; Carneiro, A.C.O.; Trugilho, P.F.; Mendes' L.M.; Carvalho, A.M.M.L. Análise termogravimétrica em clones de eucalipto como subsídio para a produção de carvão vegetal. Cerne 18 (2012) 143-151.

Savitzky, A.; Golay M.J. E. Smoothing and Differentiation of Data by Simplified Least Squares Procedures. Anal. Chem. 36 (1964) 1627-1638.

Shenk, J. S.; Workman, J. J.; Westerhaus, M. O. Application of NIR Spectroscopy to Agricultural Products. In: (3 ed.). Burns, D. A. \& Biurczak, E. W. Handbook of Near-Infrared Analysis, Florida, 2008. 